\title{
Research in Elementary Particle Physics
}

\section{TECHNICAL PROGRESS REPORT}

\author{
Lawrence J. Kirsch, Howard J. Schnitzer \\ James R Bensinger, Craig A. Blocker
}

\author{
BRANDEIS UNIVERSITY \\ Department of Physics \\ Waltham, Massachusetts 02254-9110 \\ June 1, 1991-May 31, 1992
}

\section{DISCLAIMER}

This report was prepared as an account of work. sponsored by an agency of the United States This report was prepared as an ace the United States Government nor any agency thereof, nor any of their Government. Neither the United States Government nor any agency thereof, hor assumes any legal liability or responsiemployees, makes any warranty, express of implied, or assumes any legal liability or responsibility for the accuracy, completeness, or usefulness of any information, apparatus, product, or process disclosed, or represents that its use would not infringe privately owned rights Reference herein 10 any specific commercial product, process, or service by trade name, irademark, manufacturer, or otherwise does not necessarily constitute or imply its endorsemen, recommendation, or favoring by the United States Government or any ageney thereof the of the and opinions of authors expressed herein do not

United States Government or any agency thereof.

Prepared for the United States

Department of Energy

Under DOE Contract DE-FG02-92ER40706

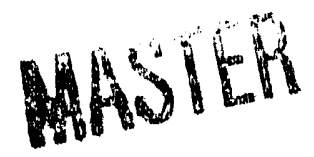




\section{TECHNICAL PROGRESS REPORT}

June 1, 1991 - May 31, 1992

Under this contract, research has been performed on both theoretical and experimental properties of elementary particles. A brief description of the work which is either in progress or has been completed is given below.

\section{EXPERIMEN'T}

During the last year the principal effort of the Brandeis High Energy Group has been directed toward the CDF detector at Fermilab. Also this year, the group began the commitment of manpower in support of the SSC program, both toward the development of the collider itself and as participants in the Solenoidal Detector Collaboration.

Brandeis officially joined the CDF collaboration in May, 1982. Prior to that we had worked informally with the CDF group at Harvard on the design of CDF detectors. We have fully participated in all of the activities of CDF including internal reviews, workshops, code development and evaluation, and assisted other members of the collaboration whenever local expertise or resources made that appropriate.

The CDF detector accumulated $4.5 \mathrm{pb}^{-1}$ of luminosity during the 1989 run which has been utilized by the Brandeis group for a series of analyses which are described below. In addition the Brandeis group has provided CDF system support in the areas of test beam, and gas detectors.

\section{CDF ANALYSIS}

\section{$B^{0}-\overline{B^{0}}$ Mixing}

The observation of a non-vanishing amount of $B^{0}-\overline{B^{0}}$ mixing by ARGUS, DESY and UA1 has been one of the more surprising observations in the last several years. Theoretical predictions of the standard model with a light top quark predict a result which is much smaller than observed, however the CDF limit of $89 \mathrm{GeV} / \mathrm{c}$ for the top quark mass call into question this last assumption.

At $\mathrm{CDF}$ the measurement is performed by determining the ratio of same sign di-electron events to the total of all di-electron events using a sample of di-electron triggers corresponding to a luminosity of $3.7 p b^{-1}$ from the 1988--89 CDF data. Since the primary $b$ quarks are produced in are produced in $b \bar{b}$ pairs, when both decay via the semileptonic channel $b \rightarrow c e \nu$, the resulting electrons will have opposite sign. The source of same sign events is either $b \rightarrow \bar{b} \rightarrow c e \nu$ (mixing), $b \rightarrow c \rightarrow e$ sequential decay, or a variety of other small backgrounds.

The first experimental problem was to determine that electrons could be reliably identified for these events since the spectrum peaks near $P_{T}$ of $2 \mathrm{GeV} / \mathrm{c}$. It had never been shown that CDF could reliably identify electrons at low $P_{T}$. From a study of pair production it was determined that criteria for electrons down to a trigger threshold of $5 \mathrm{GeV} / \mathrm{c}$ could be identified with less than $10 \%$ background. One must as well eliminate electrons from non- $b$ sources wherever possible. A number of background events contribute to opposite sign di-electrons such as photon conversions, 
$\Psi$ and $\Upsilon$ decays, and Drell-Yan events. The first two have characteristics which allow their removal without affecting the events of interest. 'The Drell--Yan is more difficult, however using a variable which measured the energy deposited in an annulus around the electron, $Z^{0} \rightarrow e^{+} e^{-}$could be used to represent the behavior of Drell-Yan events. Monte Carlo calculations were combined with a method for detecting photon conversions in order to both remove these events, and estimate the number remaining in the sample.

An extensive series of Monte Carlo events were generated using ISAJET in order to determine the ratio of direct to sequential decays, as well as to ascertain the acceptance and kinematic distributions of the various channels. Since the experimental number is a ratio, it is insensitive to the absolute $b$ cross section, but to the decay branching ratios, and the cross section for backgrourd channels. Our result for the probability of mixing is $0.172 \pm 0.065$ (statistical + systematic) \pm 0.032 (model). The result was combined with a measurement of the same processes in the $e \mu$ channel and published in Phys. Rev. Letters

\section{CDF Response to Low Energy Jets}

The substantial branching ratio for the decay of the top quark into lepton +jets has generated a sir.able interest in understanding CDF's response to low transverse energy jets $\left(E_{T}<30 \mathrm{GeV}\right)$. The definitive set of studies of low $E_{T}$ response was conducted at Brandeis. The CDF Monte Carlo detector simulation was tuned to reflect Test Beam information on response to single pions and electrons (the Test Beam pion analysis and MC tuning for the primary Central Calorimeter was also performed by Brandeis). A sample of 1.8 million Monte Carlo jet events was then generated and simulated, and analyzed to establish predictions for low $E_{T}$ jet response in the detector. Information on the absolute jet energy-scale and its systematic error was derived from this sample. Jet $E_{T}$ resolution was also studied and parametrized.

Over the last two months, the information obtained from this series of studies has been supplied to various CDF analysis efforts, including the jet multiplicity observed in $W$ events, and a. kinematic-constraint analysis of candidate top -lepton+jets events. For both these analyses, the understanding of jet $E_{T}$ systematic erro:" and resolution was crucial.

\section{Jet Scaling Behavior}

An analysis of the scaling behavior of jet production, nuw in its final stages, is being performed at Brandeis using CDF data taken at two collision energies. This analysis is receiving its final "blessing" from the CDF collaboration, and has been prepared for publication in PRL.

The hypothesis of "scaling" predicts that dimensionless jet cross-sections will be independent of $\bar{p}$ p CM energy. By contrast, perturbative QCD calculations of parton hard-scattering exhibit non-scaling behavior through the evolution of the proton structure function and the running of the strong coupling constant, $\alpha_{s}$. Previous tests of jet scaling have been limited either by large systernatic errors in comparing results from different experiments or by small lever-arm in $\sqrt{s}$ within one experiment. The present measurement utilizes CDF jet, data at the two widely-separated CM energies of 546 and $1800 \mathrm{GeV}$. We also note that in addition to providing a test of QCD predictions, the jet scaling measurement is important for extrapolating QCD event rates to LIIC and SSC energies. 
The oflline jet clustering algorithm defines jets based on calorimeter energy within a cone of radius 0.7 in $(\eta, \phi)$. Jets in this analysis are restricted to the central rapidity interval $(0.1 \leq|\eta| \leq 0.7)$. Two inclusive jet data-samples were used in this analysis: (1) the full 1988.89 run at $\sqrt{s}=1800 \mathrm{GeV}$ (integrated luminosity $\left.=4.43 p b^{-1}\right)$, and $(2)$ a short run at $\sqrt{s}=546 \mathrm{GeV}\left(8.58 n b^{-1}\right)$. Approximately $1400(40,300)$ jets in the $546(1800) \mathrm{GeV}$ sample pass all requirements.

A dimensionless cross-section can be constructed by multiplying the invariant cross-section, $E \frac{d^{3} \sigma}{d p^{3}}$, by $E_{T}^{4}$ The dimensionless cross-sections derived from the $\mathrm{CDF} \sqrt{s}=546$ and $1800 \mathrm{GeV}$ jet datasamples are expressed as functions of the dimensionless energy variable $x_{t}\left(\equiv 2 E_{T} / \sqrt{s}\right)$, and the bin-by-bin ratio of these two cross-sections is be used to test the hypothesis of scaling.

The observed inclusive jet $E_{T}$ spectra was corrected for energy loss and resolution effects. Losses result from non-linear Calorimeter response to hadrons, uninstrumented material (cracks), and non-interacting particles. Finite energy resolution introduces a smearing or "feed-up" effect to the steeply-falling jet $E_{T}$ distributions. These corrections to the measured spectra draw directly from the low $E_{T}$ jet studies described earlier. We note that the corrected inclusive cross-section $\left(\left\langle d \sigma / d E_{T}\right\rangle_{\eta}\right)$ from the 546 data-set agrees very well with previous measurements from UA1 and UA2.

We find that the ratio of dimensionless inclusive central jet cross-sections provides a test of QCD predictions with roughly twice the precision of the individual cross-sections; likewise, theoretical uncertainty is reduced by more than a factor of three in the ratio. Unlike the cross-sections, the ratio is stable under changes in jet clustering radius. Our ratio data are inconsistent with scaling (1.7\% probability of agreement), and consistent at the 1-2.3 $\sigma$ level with a range of Next-to-Leading order QCD predictions, although our data favors a level for the ratio that is lower than that of the predictions tested.

\section{Search for Pair Produced Leptoquarks at CDF}

Leptoquarks are predicted in many theories that go beyond the Standard Model. At several points in the history of physics we have been faced with complex sets of what were thought to $b$ 's the fundamental building blocks of matter and their interactions. With the advent of the quark model, the multitude of observed baryons and mesons could be described in terms of 6 more fundamental particles. These quarks, along with the 6 known leptons and the gauge bosons which mediate forces between them, constitute the Standard Model of elementary particles which we know today and which has been tested to great precision at CDF and other experiments around the world.

Despite the great success of the Standard Model we are still left with 12 apparently elementary particles. These twelve can be subdivided into two sets ( 6 quarks and 6 leptons), and furthermore each set of 6 can be divided into three groups of 2 . The Standard Model has no explanation whatsoever as to why there should be equal numbers of quarks and leptons, or why both quarks and leptons have a 3-generation doublet structure. The guark/lepton family structure symmetry, and also the collusion of leptons and quarks in canceling electro-weak anomalies naturally leads to the hypothesis that quarks and leptons are indeed related. In this larger picture quarks can be transformed into quarks, and leptons into leptons, but more importantly quarks can be transformed into leptons and vice-versa. Quark-quark and lepton-lepton transformations are already described in the standard model and are mediated via the electro-weak $W^{ \pm}$bosons. Quark-lepton transformations require a beyond-the-Standard-Model theory, and are mediated by new particles called leptoquarks. 
There are currently many contending theories of physics beyond the Standard Model. Since leptoquarks appear to be a generic feature of all these models, it is of the utmost importance that we search for them. Their discovery at CDF would be extremely important for elementary particle physics and would be the first direct evidence for post Standard Model physics.

During the past year we have completed a search for pair produced leptoquarks in the 1988-89 data set at $\operatorname{CDF}\left(4.1 \mathrm{pb}^{-1}\right)$. Leptoquarks carry color quantum numbers and so if sufficiently light would be copiously produced in $P \bar{P}$ collisions at $\sqrt{s}=1.8 \mathrm{TeV}$.

Since they decay to a lepton and a quark, there are several possible event signatures arising from the decay of a leptoquark pair. We have investigated the case where both leptons are electrons, leading to an $e^{+} e^{-}+$dijet signature.

The presence of an excess of such events above standard model backgrounds would be our first indication, but we would also be able to directly reconstruct the leptoquark mass as a well defined peak in the electron-jet mass spectrum.

The dominant backgrounds that can provide two high $P_{T}$ electrons and two jets are

- Heavy quark pair $b \bar{b}, c \bar{c}$ production followed by the semileptonic decay of both quarks

- $\gamma, Z^{0} \rightarrow e^{+} e^{-}$accompanied by a double initial state radiation.

The heavy quark background is eliminated by requiring a minimum $P_{T}$ for both leptons. We expect the number of oppositely charged dilepton events in our data set coming from $b \bar{b}$ (or $c \bar{c}$ ) decays to be negligible above a lepton $P_{T}$ threshold of $12-\mathrm{GeV}$ or so. With an electron $P_{T}$ cut of $20-\mathrm{GeV}$ we easily remove this background.

To remove the $Z^{0} \rightarrow e^{+} e^{-}+2$-jet background we cut on the invariant mass of the dielectron in the event. Using the PAPAGENO Monte Carlo event generator we have been able to estimate the differential cross section $d \sigma / d M_{e e}$ for $\left(\gamma, Z^{0} \rightarrow e^{+} e^{-}\right)+2$-jet events and so optimize our dielectron mass cut. By removing events with $75<M_{e e}<105 \mathrm{GeV}$, and requiring two $20-\mathrm{GeV}$ jets in an event, we are able to remove the background completely. After all cuts we are left with no events in our data set. The effect of the $M_{e e}$ is to reduce the signal by, at most, $30 \%$. The predictions of PAPAGENO are in very good agreement with the number of events obses ved in the data.

Requiring two $20 \mathrm{GeV}$ electrons and two $20 \mathrm{GeV}$ jets, PAPAGENO predicts 4.2 cvents background events under the $Z^{0}$ peak after all cuts, and (0.7) outside. In the data we observe $3(0)$ events respectively. Relaxing the jet $E_{T}$ cut to $15-\mathrm{GeV}$, the predictions are 8.0 (1.2) events, while we observe 8 (1) events.

In the absence of a signal we use our predictions for signal rate and cut efficiencies from the ISAJEX Monte Carlo to set limits on the Leptoquark mass and pair production cross section at CDF, and also on the leptoquark branching fraction to electron+jet. Obviously the dielectron +dijet channel is most sensitive to the case where the leptoquark decays with $100 \%$ probability to electron + jet and in this case we set our highest limit of $M_{L Q}>113 \mathrm{GeV}$ at $95 \% \mathrm{CL}$. This represents a significant improvement on the current world limit of $74 \mathrm{GeV}$ from the UA2 experiment at CERN, and in the near future will be submitted for publication in Physical Review Letters. 


\section{$B^{0}-\overline{B^{0}}$ Production Cross Section}

A measurement of the $B^{0}-\overline{B^{0}}$ production cross section is underway in the channel where both mesons decay semi-leptonically. Both the $e \mu$ and $\mu \mu$ are being studied, with the $e \mu$ channel being most promising to date. The method used to separate the $B$ 's from the background (mostly fake leptons) has been the distribution of the lepton momentum transverse to the axis of the hadronic $B$ remnants ( $P_{T}$ relative). This distribution is expected to have a higher mean value than the background due to the large $B$ mass. This distribution for the muon in the $e \mu$ sample has been fitted with a combination of a distribution from $B$ monte carlo and a fake muon distribution obtained from events triggered by a single high $P_{T}$ track. The ratio of $e \mu$ events remaining after background subtraction with like and unlike sign leptons yields a value for the $b$ mixing parameter $(\chi)$ of 0.17 . This answer is consistent with previous CDF results and will most likely be consistent with the world average once the errors have been estimated.

Future work includes similar studies of the electron $P_{T}$ relative distribution, and the use of the CLEO Monte Carlo to obtain a more accurate signal distribution.

\section{CDF SYSTEM SUPPORT}

\section{Gas Gain Calibration}

Temperature/Pressure Probes -

A. new design of temperature/pressure probe placement was implemented for the full CDF gas gain calorimetry system. This system is 20 times bigger than that at testbeam and required the calibration and installation of 240 temperature probes. These transducers are readout via communication between a $\mathrm{PC}$-based controller and the main data acquisition system, and form the database to ultimately correct the calorimetry measurements.

Gas Gain Monitor Tubes -

Brandeis constructed 100 new gas monitor calibration tubes in preparation for the 1992 run. Each tube required careful calibration under controlled conditions in a specially designed box.

\section{Test Beam}

The testbeam program ran from June to October 1991. Brandeis group members had both general shift responsibilities as well as specific tasks related is preparations for the upcoming collider run. Among these were:

High Voltage Feedback -

A new system of high roltage feedback for the gas calorimetry was being tested. This system would read the temperature and pressure throughout the gas calorimetij system and adjust the HV accordingly. Brandeis personnel calibrated and installed temperature and pressure probes, and were responsible for their readout and performance. Studies were performed on the placement of these devices to best understand temperature fluctuations throughout the detector. 


\section{SSC PROGRAM}

We have been active in the planning and design of the Solenoidal Detector Collaboration (SDC) experiment for the Superconducting SuperCollider (SSC). Professor Bensinger is a member of the SDC Technical Board which reviews the technical decisions for the whole detector, and is acting Muon Subsystem Project Manager. In this regard he has been active in the design of the muon toroidal magnet, the design of the muon measurement system, and implementation of the R\&D and engineering program to bring these designs to reality.

The barrel and forward toroidal magnets with the muon measurement system in SDC provide both a trigger on muons and identification of muons. In addition they provide a redundant measure of the muon momentum at design luminosity and will provide a robust stand alone measurement should luminosity increase substantially over design.

The barrel toroid magnet design team is completing a preliminary design and expects to hold a preliminary design review in October. The support structure for the barrel toroid has to provide adjustment for the magnet to maintain its integrity and alignment when the floor moves or during installation of the calorimeter or forward toroids. A preliminary design review is expected early next year. Due to the large size of the barrel toroid the coils are built in halves and are installed with a novel clamping arrangement.

In addition to leading the engineering effort, there have been several visits to Russia to discuss production of the magnets by Russian steel mills and to discuss the muon project with Russian physicists. We have discussed this project with engineers and production leaders from the Izhora and Atommash plants.

For the muon measurements system Professor Bensinger has been responsible for establishing the R\&D programs to develop the basic chamber design into regional designs for the various parts of the detector, the barrel, intermediate, and forward regions. In addition he has coordinated the design of the trigger and alignment systems. In this capacity he has established priorities for muon group decision making, established coordinating committees for various subsysterns of the muon system, organized and coordinated engineering meetings, and determined budgets and milestones for the group. 
"Measurement of QCD Jet Broadening in $\ddot{p} p$ Collisions at $\sqrt{s}=1.8 \mathrm{TeV}$," (with the CDF Collaboration), Phys. Rev. D 44, 601 (1991).

"A Determination of $\sin ^{2} \theta_{W}$ from the Forward-Backward Asymmetry in $p \vec{p} \rightarrow Z^{0} X \rightarrow e^{+} e^{-} X$ Interactions at $\sqrt{s}=1.8 \mathrm{TeV}$," (with the CDF Collaboration), Phys. Rev. Lett. 67, 1502 (1991).

"A Measurement of $\sigma(W \rightarrow e \nu)$ and $\sigma\left(Z^{0} \rightarrow e^{+} e^{-}\right)$in $\ddot{p} p$ Collisions at $\sqrt{s}=1800 \mathrm{GeV}$," (with the CDF Collaboration), Phys. Rev. D 44, 29 (1991).

"Measurement of the W Boson $P_{T}$ Distribution in $\bar{p} p$ Collisions at $\sqrt{s}=1.8 \mathrm{TeV}$," (with the CDF Collaboration), Phys. Rev. Lett. 66, 2951 (1991).

"Measurement of the $Z p_{T}$ Distribution in $\bar{p} p$ Collisions at $\sqrt{s}=1.8 \mathrm{TeV}$," (with the CDF Collaboration), Phys. Rev. Lett. 67, 2937 (1991).

"Measurement of the $e^{+} e^{-}$Invariant Mass Distribution in $\bar{p} p$ Collisions at $\sqrt{s}=1.8 \mathrm{TeV}$," (with the CDF Collaboration), Phys. Rev. Lett. 67, 2418 (1991).

"Lepton Asymmetry in W Decays from $\bar{p} p$ Collisions at $\sqrt{s}=1.8 \mathrm{Tev}$," (with the CDF Collaboration), Phys. Rev. Lett. 68, 1458 (1992).

"Topology of Three Jet Events in $\bar{p} p$ Collisions at $\sqrt{s}=1.8 \mathrm{TeV}$," (with the CDF Collaboration), Phys. Rev. D 45, 1448 (1992).

"Inclusive Jet Cross Section in $p \bar{p}$ Collisions at $\sqrt{s}=1.8 \mathrm{TeV}$," (with the CDF Collaboration), Phys. Rev. Lett. 68, 1104 (1992).

"Inclusive Jet Cross Section in $p \bar{p}$ Collisions at $\sqrt{s}=1.8 \mathrm{TeV}$," (with the CDF Collaboration), to be submitted to Phys. Rev. D.

"Search for $W^{\prime} \rightarrow e \nu$ and $W^{\prime} \rightarrow \mu \nu$ in $\bar{p} p$ Collisions at $\sqrt{s}=1.8 \mathrm{TeV}$," (with the CDF Collaboration), Phys. Rev. Lett. 67, 2609 (1991).

"Measurement of $B^{\circ} \bar{B}^{\circ}$ Mixing at the Fermilab Tevatron Collider," (with the CDF Collaboration), Phys. Rev. Lett. 67, 3351 (1991).

"Measurement of the Ratio $\sigma B(W \rightarrow \tau \nu) / \sigma B(W \rightarrow e \nu)$ in $p \bar{p}$ Collisions at $\sqrt{s}=1.8 \mathrm{TeV}$, as a T'est of Lepton Universality," (with the CDF Collaboration), submitted to Phys. Rev. Lett., December $23,1991$.

"A Lower Limit on the Top Quark Mass from Events with Two Leptons in $p \bar{p}$ Collisions at $\sqrt{s}=$ $1.8 \mathrm{TeV}$," (with the CDF Collaboration), Phys. Rev. Lett. 68, 447 (1992).

"Search for Squarks and Gluinos from $p \bar{p}$ Collisions at $\sqrt{s}=1.8 \mathrm{TeV}$," (with the CDF Collaboration), to be submitted to Phys. Rev. Lett.

"Properties of Events with Large Total Transverse Energy Produced in Proton-Antiproton Collisions at $\sqrt{s}=1.8 \mathrm{TeV}$," Phys. Rev. D 45, 2249 (1992). 
"A Search for New Gauge Bosons in $\bar{p} p$ Collisions at $\sqrt{s}=1.8 \mathrm{TeV}$," (with the CDF Collaboration), Phys. Rev. Lett. 68, 1463 (1992).

"A Limit on the Top Quark Mass from Proton-Antiproton Collisions at $\sqrt{s}=1800 \mathrm{GeV}$," (with the CDF Collaboration), submitted to Phys. Rev. D., December 19, 1991.

"A Measurement of the $B$ Meson and $b$ Quark Cross Section at $\sqrt{s}=1.8 \mathrm{TeV}$ Using the Exclusive Decay $B^{ \pm} \rightarrow J / \psi K^{ \pm}$," (with the CDF Collaboration), submitted to Phys. Rev. Lett., January 24, 1992.

"Measurement of the Isolated Prompt Photon Cross Section Measurement in $p \bar{p}$ Collisions at $\sqrt{s}$ $=1.8$ 'TeV," (with the CDF Collaboration), submitted to Phys. Rev. Lett., February 3, 1992.

"A Measurement of the Production and Muonic Decay Rate of $W$ and $Z$ Bosons in $p \bar{p}$ Collisions at $\sqrt{3}=1.8 \mathrm{TeV}$," (with the CDF Collaboration), submitted to Phys. Rev. Lett., February 25, $19 \iota 2$.

"A Measurement of the Bottom Quark Production in 1.8-TeV Proton-Antiproton Collisions using Semileptonic Decay Electrons," (with the CDF Collaboration), to be submitted to Phys. Rev. Lett.

"Limit on the Rare Decay $W^{ \pm} \rightarrow \gamma+\pi^{ \pm}$in $p \bar{p}$ Collisions at $\sqrt{s}=1.8 \mathrm{TeV}$," (with the CDF Collaboration), to be subrnitted to Phys. Rev. Lett.

"A Measurement of Jet Shapes in $\bar{p} p$ Collisions at $\sqrt{s}=1.8 \mathrm{TeV}$," (with the CDF Collaboration), to be submitted to Phys. Rev. Lett.

"Limits on the Production of Massive Stable Charged Particles," (with the CDF Collaboration), to be submitted to Phys. Rev. D, Rapid Communications.

"The Dijet Angular Distribution at $\sqrt{s}=1.8 \mathrm{TeV}$," (with the CDF Collaboration), to be submitted to Phys. Rev. Lett. 


\section{THEORY}

During the past year, the theoretical physics group pursued research in quantum field theory and neural networks. This represents the work of Prof. Schnitzer, Drs. Bourdeau and Riggs, and Mr. Mlawer for the entire year, that of Prof. Abbott from June 1, 1991 to December 31, 1991, as well as that of Dr. Sergei Pokrovsky, who was an unpaid visitor to the group for the entire contract year.

\section{QUANTUM FIELD THEORY}

\section{Lie Superalgebras in Chern-Simons and WZW Theory}

The Lie superalgebras $S U(m \mid n)$ are currently receiving renewed attention in the context of twodimensional current algebra and three-dimensional Chern-Simons theory. While the SU(m/n) current algebra has been considered, the structure of the $S U(m \mid n) W Z W$ operator (or fusion) algebras had been left unexamined. Bourdeau, Mlawer, Riggs, and Schnitzer found a simple description of the $\mathrm{SU}(\mathrm{n}+\mathrm{N} \mid \mathrm{n})_{\mathrm{K}}$ fusion algebra in terms of that of the Kac-Moody algebra $\mathrm{SU}(\mathrm{N})_{K}$. It was found that the existence and structure of a subring of positive $q$-superdimensions characterizes the $\mathrm{SU}(\mathrm{m} \mid \mathrm{n})_{\mathrm{K}}$ fusion ring in the same way that the subring of positive $q$-dimensions characterizes the $\mathrm{SU}(\mathrm{N})_{\mathrm{K}}$ fusion ring.

The analysis proceeds by meaus of a combined examination of the ring of positive $q$-superdimensions, associated Chern-Simons observables, and several (Wess-Zumino-Witten) WZW correlation functions. The $S U(m \mid n)_{K}$ WZW models were found to be quasi-rational (nonunitary) conformal field theories. Many important quantities in $\mathrm{SU}(\mathrm{m} \mid \mathrm{n})_{\mathrm{K}}$ theories are given exactly by analogous $\mathrm{SU}(\mathrm{m}-$ $\mathrm{n})_{\mathrm{K}}$ quantities, including the conformal weights, fusion coefficients, and Chern-Simons observables. The $\mathrm{SU}(\mathrm{n}+\mathrm{N} \mid \mathrm{n})_{\mathrm{K}}$ and $\mathrm{SU}(\mathrm{k}+\mathrm{K} \mid \mathrm{k})_{\mathrm{N}}$ Chern-Simons and $\mathrm{WZW}$ theories are related by the group-level duality previously discovered, and extensively analyzed by our group.

\section{Topological Field Theory}

From the beginning the Landau-Ginzburg (LG) approach has provided a useful picture of rational conformal field theories. More recently the LG formulation of $\mathrm{N}=2$ superconformal theories has yielded detailed non-perturbative results, since the equation of motion for the superpotential $d W=$ 0 are not subject to renormalizations. A subset of the field in such LG theories form a closed, non-singular operator algebra, the chiral ring. Such field have the property that their correlation functions are topological. The non-topological fields can be vemoved by twisting these theories to obtain LG models which are entirely topological. It is remarkable that there are deformations of the $N=2$ superconformal theories to non-conformal (but still $N=2$ supersymmetric) theories which retain a closed topological ring.

A particularly interesting example, a set of LG potentials whose chiral rings are isomorphic to the (non-conformal) $\mathrm{SU}(\mathrm{N})_{\mathrm{K}}$ WZW fusion rings, was presented by Gepner. Bourdeau, Mlawer, Riggs, and Schnitzer gave the first description of a fusion ring of a non-simply laced group $\mathrm{Sp}(\mathrm{N})_{\mathrm{K}}$ as a (non-conformal) LG ring. They showed that the $\mathrm{SU}(\mathrm{N}+1)_{\mathrm{K}}$ and $\mathrm{Sp}(\mathrm{N})_{\mathrm{K}}$ fusion rings are two different deformations of the same conformal theory. A further result was that the twisted versions of the $\mathrm{Sp}(\mathrm{N})_{\mathrm{K}}$ and $\mathrm{Sp}(\mathrm{K})_{\mathrm{N}}$ topological LG theories are identical. They also found that although 
the $\mathrm{SU}(\mathrm{N})_{\mathrm{K}}$ and $\mathrm{SU}(\mathrm{K})_{\mathrm{N}}$ theories are not identical, their correlation functions are closely related. Therefore, this extends the concept of group-level duality to topological LG field theories.

\section{Group-Level Duality for Chern-Simons Observables}

Topologically invariant Chern-Simons gauge theories in $2+1$ dimensions are interacting, yet soluble, quantum field theories. Quantization of such a theory with compact gauge group $G$ forces the coupling constant $\mathrm{K}$ to be an integer. The fixing of these integer values of $\mathrm{K}$ leads to the appearance of discrete symmetries associated to automorphisms of the extended Dynkin diagram for $G$, as well as duality relations between models with classical group $G(N)$ as gauge group and coupling constant $K$ and those with gauge group $G(K)$ and coupling constant $N$. That is, the group-level duality $\mathrm{G}(\mathrm{N})_{\mathrm{K}} \rightarrow \mathrm{G}(\mathrm{K})_{\mathrm{N}}$ discovered by the Brandeis group appears in this context as well.

Arbitrary gauge invariant observables of Chern-Simons theories correspond to linked-often knottedWilson lines and graphs. These observables can then be reduced to sums of products of tetrahedra graphs. Naculich, Riggs, and Schnitzer showed that such tetrahedral observables fall into sets related by the Dynkin diagram automorphisms, and have in addition rank-level duals in the $G(K)_{N}$ theory. They also obtained the transformation properties of arbitrary graphs, knots, and links under these Dynkin diagram automorphisms, as well as under group-level duality. Therefore, this quantum generated symmetry has important implications for the observables of Chern-Simons theories.

\section{Double Scaling Limit in $\mathbf{O}(\mathrm{N})$ Theory in Four-Dimensions}

The double scaling limit allows for new, non-perturbative insights into the phase structure of quantum field theories. An essential feature of these methods is the existence of a critical value of a coupling constant $g_{c}$, and a double scaling limit $N \rightarrow \infty$ and $g \rightarrow g_{c}$, such that an appropriate correlated scaling variable is kept fixed. For the most part, the approach has been applied to $c \leq 1$ matter coupled to two-dimensional gravity. However, other field theories which allow for a double scaling limit have also been considered. In particular the $O(N)$ vector model and generalized vector models have been studied in $\mathrm{D}=0,1,2,3$ and 4 dimensions by Zinn-Justin and by Di Vecchia, Kato, and Ohta.

Schnitzer reexamined the double scaling limit for $\left(\Phi^{2}\right)^{2} O(N)$ theory in $D=4$ dimensions. He showed that precisely at the critical point the effective potential for the $\mathrm{D}=4$ theory becomes everywhere complex, which undermines the consistency of the $D=4 \mathrm{O}(\mathrm{N})$ vector model at the critical point, and thus makes the existence of a double scaling limit doubtful. This probler. does not arise for $\mathrm{D}<4$.

\section{Sine-Gordon Theory}

Pokrovsky applied a quantum group analysis to the Sine-Gordon theory in a strong-coupling region. Infinitely many bound states were found together with the corresponding S-matrices. These new solutions of the Yang-Baxter equations are related to some reducible representations of the quantum $s \ell(2)$ algebras, resembling the Kac-Moody algebra representations of WZW theory. 


\section{NEUFAL NETWORKS}

Abbott, Farhi, and Gutmann constructed the path integral for determining the potentiai of any dendritic tree described by a linear cable equation. This was done by generalizing Brownian motion from a line to a tree. They also constructed the path integral for dendritic structures with spatiallyvarying and/or time-dependent membrane conductivities due, for example, to synaptic inputs. The path integral allows novel computational techniques to be applied to cable problems. Their analysis leads to an exact expression for the Green's function of a dendritic tree of arbitrary geometry expressed in terms of a set of simple diagrammatic rules. These rules provide a fast and efficient method for solving complex cable problems.

Abbott derived an expression relating the input current for a single neuron in a neural network to the firing rates of excitatory and inhibitory inputs synapsing on the dendritic tree of the neuron. Any dendritic geometry and any pattern of synaptic connection can be treated using these techniques. The input currents caiculated, combined with known firing rate functions, allow the effects of synaptic conduct:unce changes along dendritic cables to be included in a mean-field description of network behavior. The shunting effects of inhibitory synaptic conductances provide a solution to the high firing rate problem in neural network models.

Abbott discussed the construction of models that describe the firing rates of excitatory and inhibitory neurons in biological neural networks.

A model was presented that incorporated both slow linear and fast nonlinear inhibition. With the appropriate excitatory-to-excitatory couplings the model can act as an associative memory in which pattern recognition is signalled by resonant firing behavior. Stored memories are represented by fixed points of the excitatory and fast inhibitory dynamics. After memory recovery, slow inhibition returns the system to the silent, resting state.

Van Vreeswijk and Abbott analyzed a coupled population of simple integrate and fire model neurons to determine the conditions that lead to stable firing sustained by all-to-all excitatory interactions. The possible temporal firing patterns were determined and the initial conditions that produce them were discussed. For certain parameter values two different patterns of firing are possible, one in which the firing is distributed among many groups of neurons that fire sequentially, and another in which the population breaks into two groups that fire alternately. The probabilities of different temporal firing patterns arising from random initial conditions and noise inputs were analyzed by computer simulation. 


\section{QUANTUM FIELD THEORY}

1. "The Quasi-Rational Fusion Structure of SU(m|n) Chern-Simons and WZW Theories," (Bourdeau, Mlawer, Riggs, and Schnitzer), Nucl. Physics B372, 303-358 (1992).

2. "Topological Landau-Ginsburg Matter from $S_{2}(\mathrm{~N})_{K}$ Rusion Rings" (Bourdeau, Mlawer, Riggs, and Schnitzer), Mod. Phys. Lett. A.7, 689 (1992).

3. "Simple-Current Symmetries, Rank-Level Duality, and Linear Skein Relations for ChernSimons Graphs" (Nwculich, Riggs, and Schnitzer), Brandeis preprint BRX-XH-302 (57 pagea).

4. "Concerning the Double Scaling Limit in the $O(N)$ Vector Model in Four-Dimensions," (Schnitzer) Brandeis preprint BRX.TH-333 (5 pages).

5. "When Can Identical Particles Collide?" (Bourdeau ans! Sorkin), Phys. Rev. D45, 687-696 (1992).

6. "Quartum Group Analysis of the Bound States in the Strong Coupling Regime of the Modified Sine--Gordon Model," (Pokrovsky), preprint (28 pages').

\section{NEURAL NETWORKS}

7. "Realistic Synaptic Inputs for Model Neural Networks" (Abbott), Network 2, 245-258 (1991).

8. "The Path Integral for Dentritic Trees" (Abbott, Farhi, and Gutmann), Biol. Cybern. 66, $49-60(1991)$.

9. "Simple Diagrammatic Rule: for Solving Dendritic Cable Problems" (Abbott), Physica A (to be published) (16 pages).

10. "Self-Sustained Firing in Populations of Integrate and Fire Neurons" (Abbott and Van Vreeswijk), SIAM Journ. of Appl. Math. (to be published) (19 pages).

11. "Firing-Rate Models for Neural Populations" (Abbott), to appear in the Proceedings of the Conference on Neural Networks: From Biology to High-Energy Physics (17 pages). 


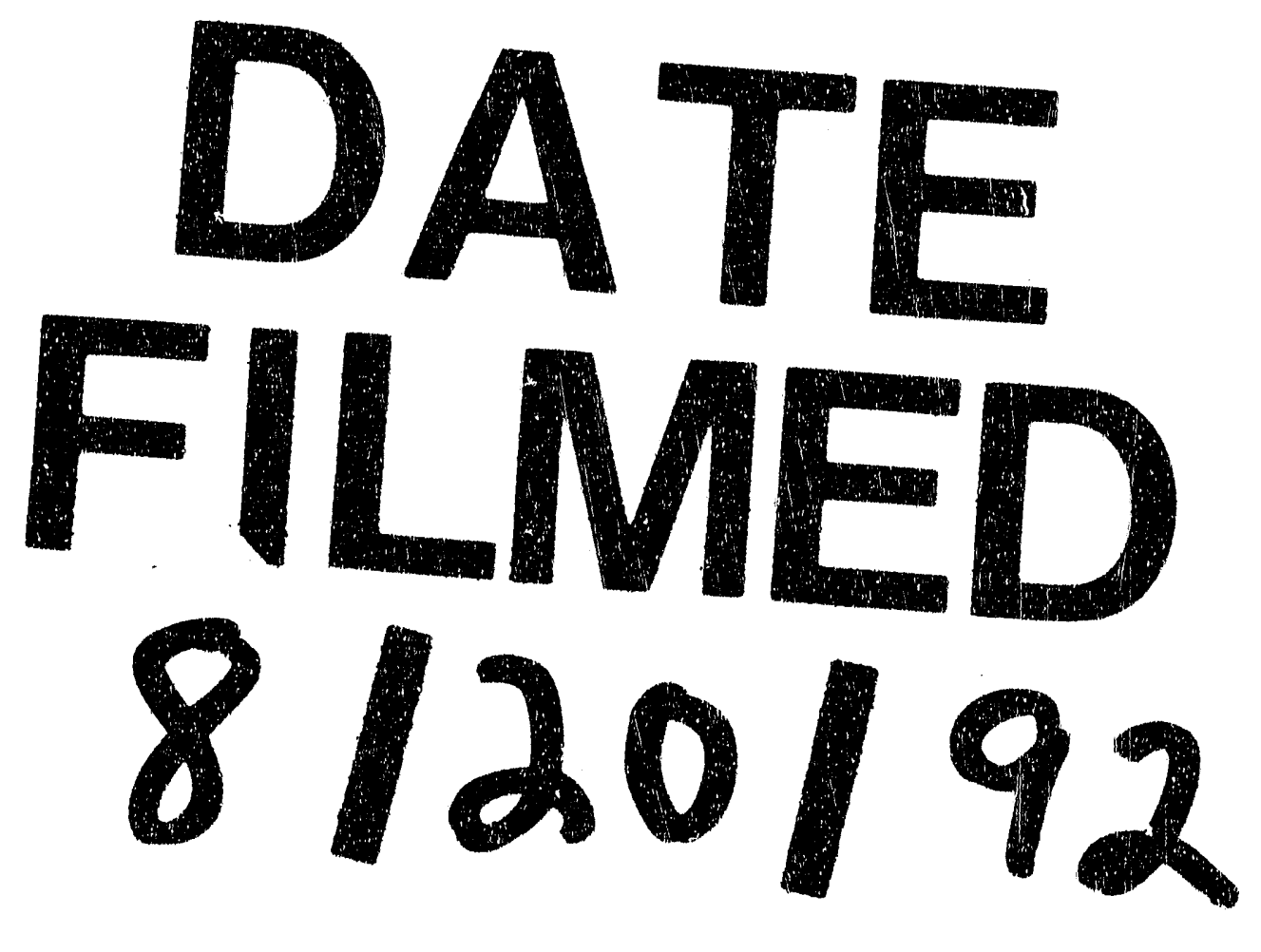


